\title{
THE APPLICATION OF THE BrilLanCe SERIES SCINTILLATION DETECTOR IN THE SPECTROMETER OF NEUTRAL PARTICLES FOR THE SATELLITE EXPERIMENT ZINA-NT
}

\author{
IRENE V. ARKHANGELSKAJA*, ANDREY I. ARKHANGELSKY, \\ ALEXEY.G BATISCHEV, ARKADY M. GALPER \\ Department of Experimental Nuclear Physics and Cosmophysics, \\ National Research Nuclear University "MEPhI", Kashirskoe shosse, \\ 31 Moscow, 115409, Russia \\ *irene.belousova@usa.net \\ NATALYA V. KONDRATYEVA, PETR YU. NAUMOV \\ Department of Experimental Methods in Nuclear Physics, \\ National Research Nuclear University "MEPhI", Kashirskoe shosse, \\ 31 Moscow, 115409, Russia
}

\begin{abstract}
Spectrometric detector complex ZINA-NT is intended to study a radiation conditions onboard satellite and characteristics of hard X-ray and gamma-ray fluxes from GRB, solar flares and to detect other non-stationary fluxes of cosmic gamma-rays. The advantages for using of this new detector for modification of present neutral particles detector on the base of $\mathrm{CsI}(\mathrm{Tl})$ are discussed. Scintillation detectors based on BrilLanCe series crystal have got very small lighting time, an excellent energy resolution and light output, more intensive than devices based on $\mathrm{CsI}(\mathrm{Tl})$. Using of BrilLanCe series crystal instead of $\mathrm{CsI}(\mathrm{Tl})$ ones allows to detect terrestrial gamma flashes (TGF), gamma ray bursts (GRB) and solar flares with smaller time durations and intensities. Moreover, the counts rate linearity region of BrilLanCe detectors is extended up to $10^{6} s^{-1}$ and it allows to observe a very intensive events. So, the using of such type of detector permits us the possibility to separate gamma-quanta and neutrons on the timescales less than 50 microseconds.
\end{abstract}

Keywords: Pulse-shape discrimination; BrilLanCe series scintillators; detectors for satellite experiments.

\section{Introduction}

Spectrometric complex of scientific instruments ZINA-NT consists of multilayer scintillation spectrometer of charged particles (MSS) ${ }^{1,2}$ and detector of neutral particles $(\mathrm{DNP})^{3}$. Data acquisition and registration of scientific information system $(\mathrm{SSOI})^{4,5}$ is part of multilayer scintillation spectrometer but used for detector of neutral particles too.

The tasks of the experiment will be space weather and magnetospheric particles flows monitoring, studying flows of charged particles and neutrons during solar flares, investigation of X-ray and $\gamma$-emission up to $300 \mathrm{MeV}$ during solar flares 
(including 15.11 MeV and 20.58 MeV lines), Gamma Ray Bursts (GRB) and Terrestrial Gamma Flashes (TGF). Also DNP detector will allows detailed investigation of registered events energy spectra in wide band.

In 2011 it is planned to create experimental sample of apparatus. The experiment ZINA-NT will be installed onboard "Meteor-M" satellite which will be launched in 2015 .

\section{ZINA-NT apparatus short description}

Multilayer Scintillation Spectrometer of charged particles (MSS) includes Multilayer Scintillation Detector (MSD) with high speed amplifiers and data acquisition and registration of scientific information system (SSOI). Main parameters of MSD detector and its outward view are presented in Table 1 and Fig 1 a respectively. MSD consists of ten plates (C1-C10) from polystyrene scintillator (each of the top two plates divides by four strips) with different size viewed by 24 photomultipliers. Top three plates produce the trigger signal and plates from 3 to 9 are used as charged particles calorimeter. Bottom plate works as anticoincidence shield with efficiency better then 0.99 .

The construction and the function diagram of DNP are presented on Fig 1b and Fig 2. This device consists of spectrometric detector C11 on the base of crystal scintillator for hard X-ray and gamma-ray analysis fully surrounded by plastic anticoincidence shield (detectors C12-C14). This complex of detectors viewed by three groups of photomultipliers. Registration efficiency of anticoincidence shield is about 0.999 for reliable neutral particles identification. For this system trigger will be:

$$
M S T=(\overline{A C}) A N D \mathrm{C} 11,
$$

where

$$
A C=\left(\begin{array}{llllllll}
\mathrm{C} 14 & \text { OR } & \mathrm{C} 13 & \text { OR } & \mathrm{C} 12-1 & \text { OR } & \mathrm{C} 12-2 & \text { OR } \\
\mathrm{C} 12-3 & \text { OR } & \mathrm{C} 12-4
\end{array}\right) .
$$

Electronics of DNP contains of preamplifier-shaper for triggering the detector and spectrometry system for neutral particles identification. The discrimination between neutron and $\gamma$-ray events is performed using the pulse-shape discrimination technique for the light pulse emitted by a scintillation detector based on the

Table 1. The main parameters of MSD (preliminary).

\begin{tabular}{lc}
\hline Parameter & Value \\
\hline Energy bands: & \\
Electrons and positrons & $4-25 \mathrm{MeV}$ \\
Protons and He nuclei & $40-110 \mathrm{MeV} /$ nucleon \\
Energy resolution: & $1,5-4 \%$ \\
Protons and He nuclei & $6-8 \%$ \\
Electrons & $40 \mathrm{~cm}^{2} \times \mathrm{sr}$ \\
Geometric quotient & $80^{\circ}$ \\
Aperture & $100 \mathrm{~ns}$ \\
Time resolution & \\
\hline
\end{tabular}



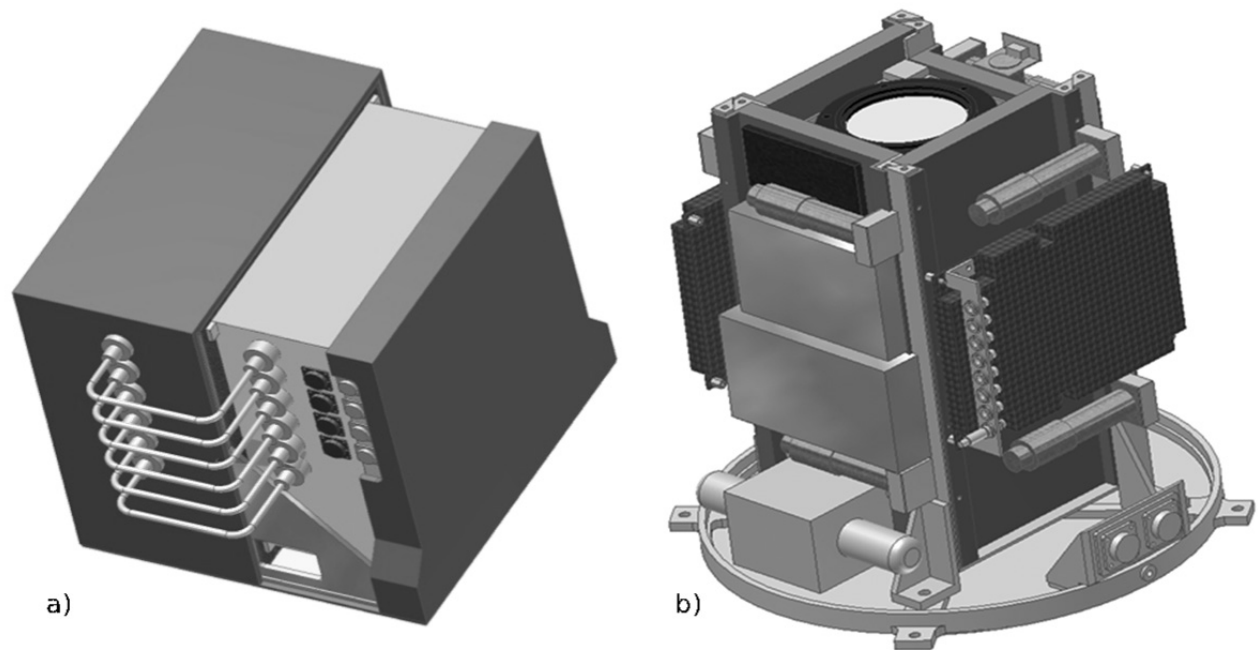

Fig. 1. The construction of the detectors MSD (a) and DNP (b)

dependence of the ratio of the light-output components intensities, with different fluorescence decay times, on the average ionization density produced by charged particles in the detector material ${ }^{6}$.

Previously we plane to use $\mathrm{CsI}(\mathrm{Tl})$ crystal with diameter $76 \mathrm{~mm}$ and $76 \mathrm{~mm}$ height. When neutrons with energies of $\sim 5-10^{3} \mathrm{MeV}$ interact with $\mathrm{Cs}$ and I nuclei, highly ionizing particles are produced: hydrogen and helium isotopes with average energies of $\sim 10$ and $\sim 15-20 \mathrm{MeV}$, respectively. As a result of $\gamma$-quanta

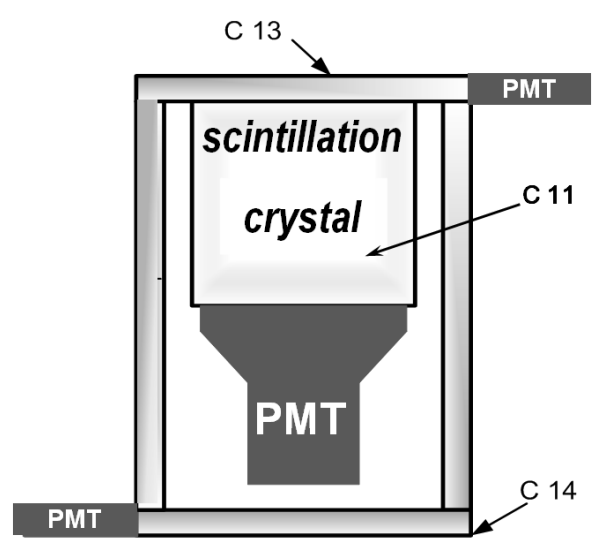

a)

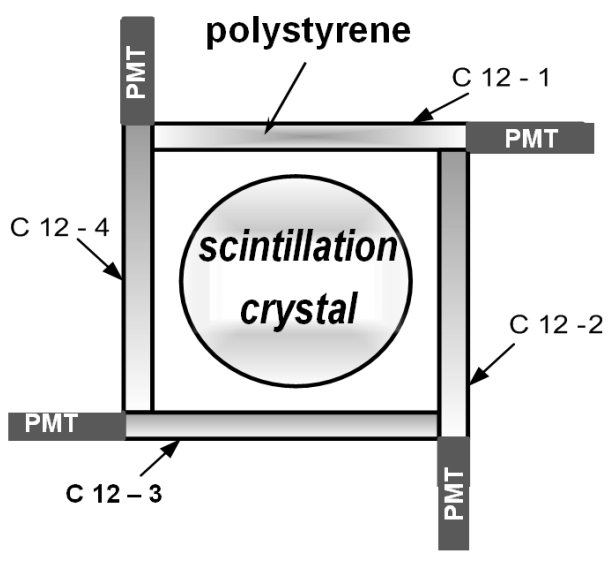

b)

Fig. 2. The function diagram of the detector DNP: front view (a) and top view (b) 
interactions, electrons and positrons are generated, for which the specific ionization produced in the substance at energies of several hundreds $\mathrm{keV}$ and higher is close to its minimum. A scintillation flash in $\mathrm{CsI}(\mathrm{Tl})$ consists of two main fluorescence components with decay times $\tau_{\text {fast }} \sim 0.4-0.7 \mu$ s and $\tau_{\text {slow }} \sim 7 \mu \mathrm{s}$, and the ratio of the slow component intensity $\mathrm{Q}_{\text {slow }}$ to the fast component intensity $\mathrm{Q}_{\text {fast }}$ depends on the specific ionization of the interacting particles. This ratio changes from $\sim 1$ for relativistic particles down to $\sim 0.25$ for $\alpha$-particles with energies of $\sim 10 \mathrm{MeV}$.

Information from DNP is received by SSOI MSS and translates to onboard satellite telemetry system.

Both detectors now developed in National Research Nuclear University "MEPhI" and SSOI system is constructed in Physics Institute of National Academy of Science of the Republic of Belalus.

\section{Comparison of BrilLanCe series crystal with wide used scintillators of other types}

Scintillator class BrilLanCe was used firstly in Delft University (Netherland). These scintillators are produced by SAINT GOBAIN CERAMICS \& PLASTICS. In this work two types of such crystal will be inspected: BrilLanCe350 $\left(\mathrm{LaCl}_{3}\right.$ : Ce $(10 \% \mathrm{Ce}))$ and BrilLanCe380 $\left(\mathrm{LaBr}_{3}: \mathrm{Ce}(5 \% \mathrm{Ce})\right)$.

The main characteristics comparison of $\mathrm{NaI}(\mathrm{Tl}), \mathrm{CsI}(\mathrm{Tl})$ and BrilLanCe crystals (all have got at least two light output components) is presented in Table 2 (compilation from ${ }^{7}$ ). The temperature dependence of relative pulse height for different scintillators is shown on the Fig 3. It is seen, that the most week dependence of pulse height on temperature is for crystals B350 (BrilLanCe350) and B380 (BrilLanCe380) in the ranges $25-125^{\circ} \mathrm{C}$ and $25-175^{\circ} \mathrm{C}$ correspondingly. Also more

Table 2. The main characteristics of $\mathrm{NaI}(\mathrm{Tl}), \mathrm{CsI}(\mathrm{Tl})$ and BrilLanCe crystals.

\begin{tabular}{|c|c|c|c|c|}
\hline Characteristics & $\mathrm{NaI}(\mathrm{Tl})$ & $\mathrm{CsI}(\mathrm{Tl})$ & $\mathrm{LaBr}_{3}: \mathrm{Ce}$ & $\mathrm{LaCl}_{3}: \mathrm{Ce}$ \\
\hline Light output, quanta/keV & 38 & 45 & 65 & 48 \\
\hline Fast time $(\mathrm{tf}), \mathrm{ns}$ & 220 & 700 & 18 & 25 \\
\hline Slow time $(\mathrm{ts}), \mu \mathrm{s}$ & 1.4 & $2-5$ & 0.8 unstable & 0.5 \\
\hline $\begin{array}{l}\text { Relation between components } \\
\left(\mathrm{C}_{\mathrm{f}} \text { ast } / \mathrm{C}_{\mathrm{s}} \text { low }\right) \text { at } 25^{\circ} \mathrm{C}, \%\end{array}$ & $85: 15$ & $55: 45$ & $90: 10$ & $80: 20$ \\
\hline $\begin{array}{l}\text { Fluorescence from high energy } \\
\text { charged particles }\end{array}$ & weakly & up to $20 \mathrm{~ms}$ & weakly & weakly \\
\hline Density, $\mathrm{g} / \mathrm{sm}^{3}$ & 3.67 & 4.51 & 5.08 & 3.85 \\
\hline $\begin{array}{l}\lambda, \mathrm{nm} \\
\text { (at which light output is } \max \text { ) }\end{array}$ & 410 & 565 & 380 & 350 \\
\hline Mechanical stress resistance & low & high & high & high \\
\hline Energy resolution $(662 \mathrm{keV})$ & $7 \%$ & $9 \%$ & $2,9 \%$ & $3,8 \%$ \\
\hline Water resistance & yes & no & weakly & $\begin{array}{c}\text { weakly } \\
\text { (at high } T^{o} \text { ) }\end{array}$ \\
\hline Radiation hardness, rad & $10^{5}$ & $10^{7}$ & $1,5 \times 10^{7}$ & $10^{7}$ \\
\hline
\end{tabular}




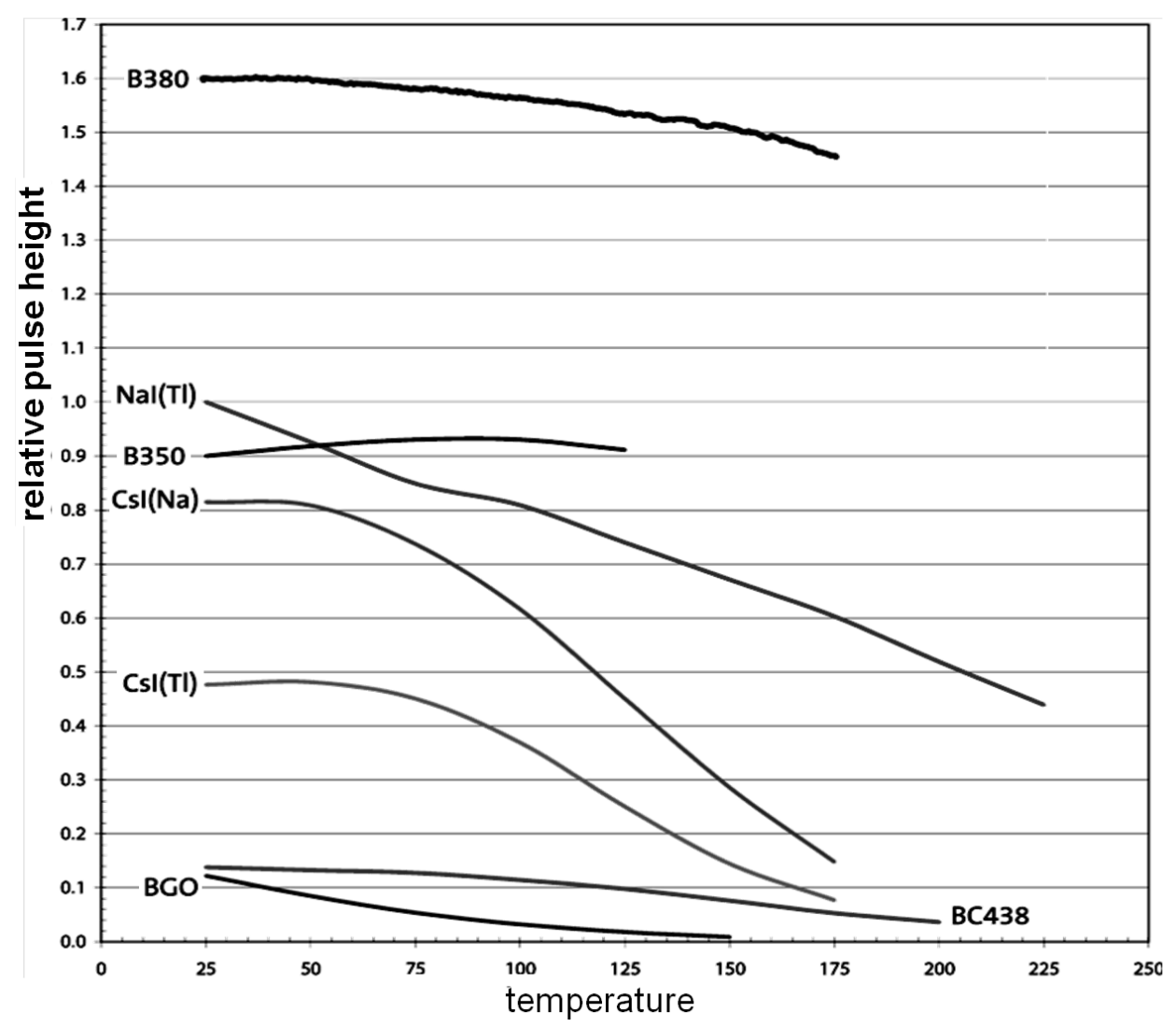

Fig. 3. The temperature dependence of relative pulse height for different scintillators. Compilation from ${ }^{7}$.

or less linearity regions occur for scintillators $\mathrm{CsI}(\mathrm{Tl}), \mathrm{CsI}(\mathrm{Na})$ and $\mathrm{BC} 438$ in the bands $25-70^{\circ} \mathrm{C}, 25-55^{\circ} \mathrm{C}$ and $25-120^{\circ} \mathrm{C}$ respectively.

But from duscussed scintillators only three of them have got several light output components with different timescales (see Table 2) and could be used for sorting of the particles with different ionizing capability by the pulse shape analyzing method. Unfortunately second component in the B380 crystal is unstable and it leads to some difficulties with particles identification in wide temperature band. The detailed shapes of pulses on the input of $\mathrm{ADC}$ for $\mathrm{CsI}(\mathrm{Tl})$ and $\mathrm{B} 350$ crystals are shown at the Fig 4.

The possibility of particles discrimination with proper accuracy by detailed analysis of light pulse shape using BrilLanCe series scintillators discussed in some works ${ }^{8,9}$ but in this case it is necessary to use very high speed digital ADC (because of very short pulse duration). 

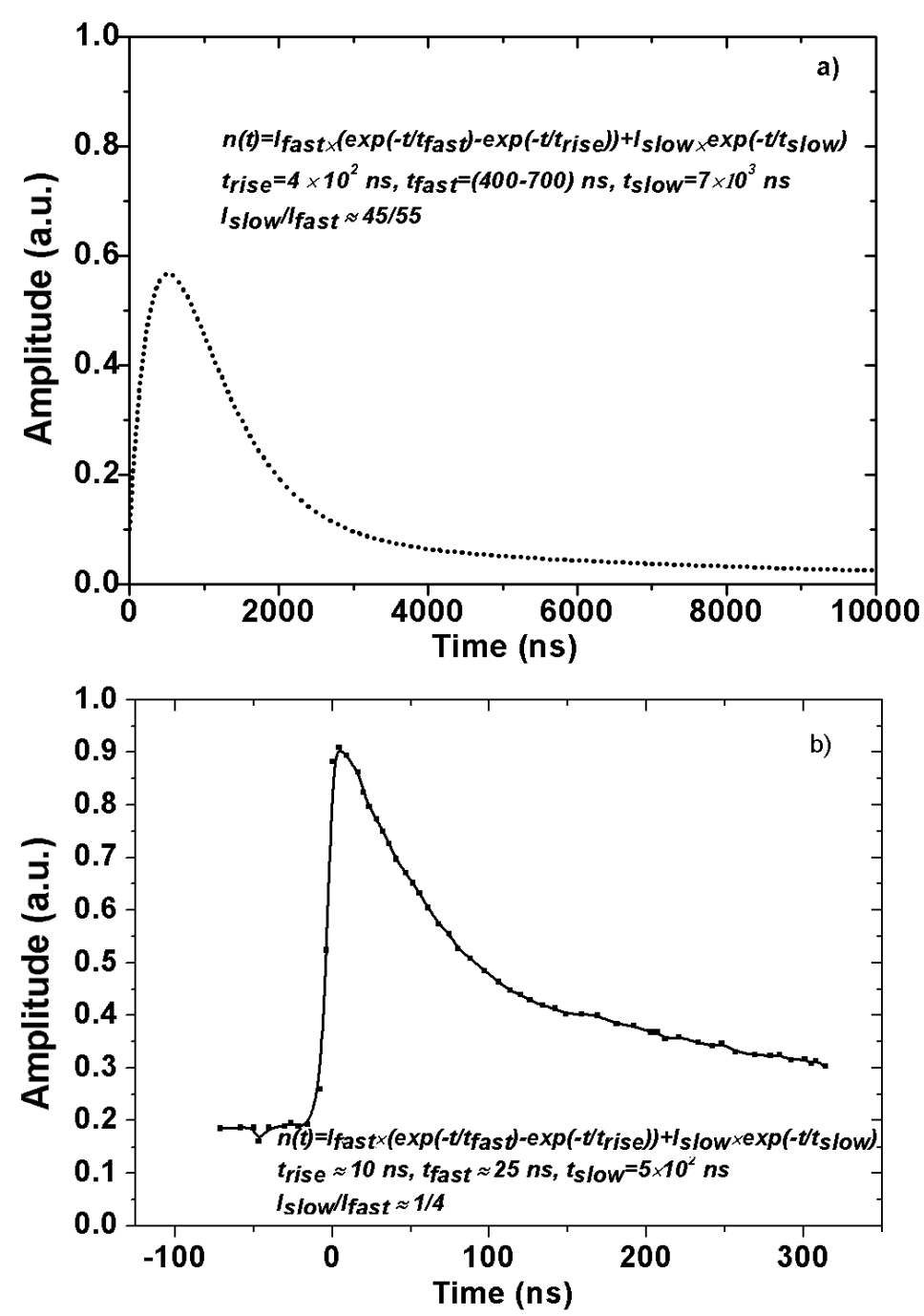

Fig. 4. The detailed shapes of pulses on the input of ADC for the crystals: a) $\operatorname{CsI}(\mathrm{Tl})$ and b) B350 (adopted from ${ }^{10}$ ).

The energy spectra from the radioactive source ${ }^{137} \mathrm{Cs}\left(\mathrm{E}_{\gamma}=662 \mathrm{keV}\right)$ in the $\mathrm{CsI}(\mathrm{Tl})$ and B350 scintillators are presented at the Fig 5. Energy resolution of the combination of 3 " $\times 3$ " CsI $(\mathrm{Tl})$ crystal with PMT FEU-110 is $(\Delta \mathrm{E} / \mathrm{E})_{\mathrm{CsI}(\mathrm{Tl})} \sim 13 \%$ but one of the combination of 3" $\times 3$ " B350 crystal with PMT EMI9265-B53/FL is more than twice better and $(\Delta \mathrm{E} / \mathrm{E})_{\mathrm{B} 350} \sim 5.4 \%$. The energy resolution of this crystal is slightly worse than $3.8 \%$ according to Saint Gobain data ${ }^{12}$. This fact 

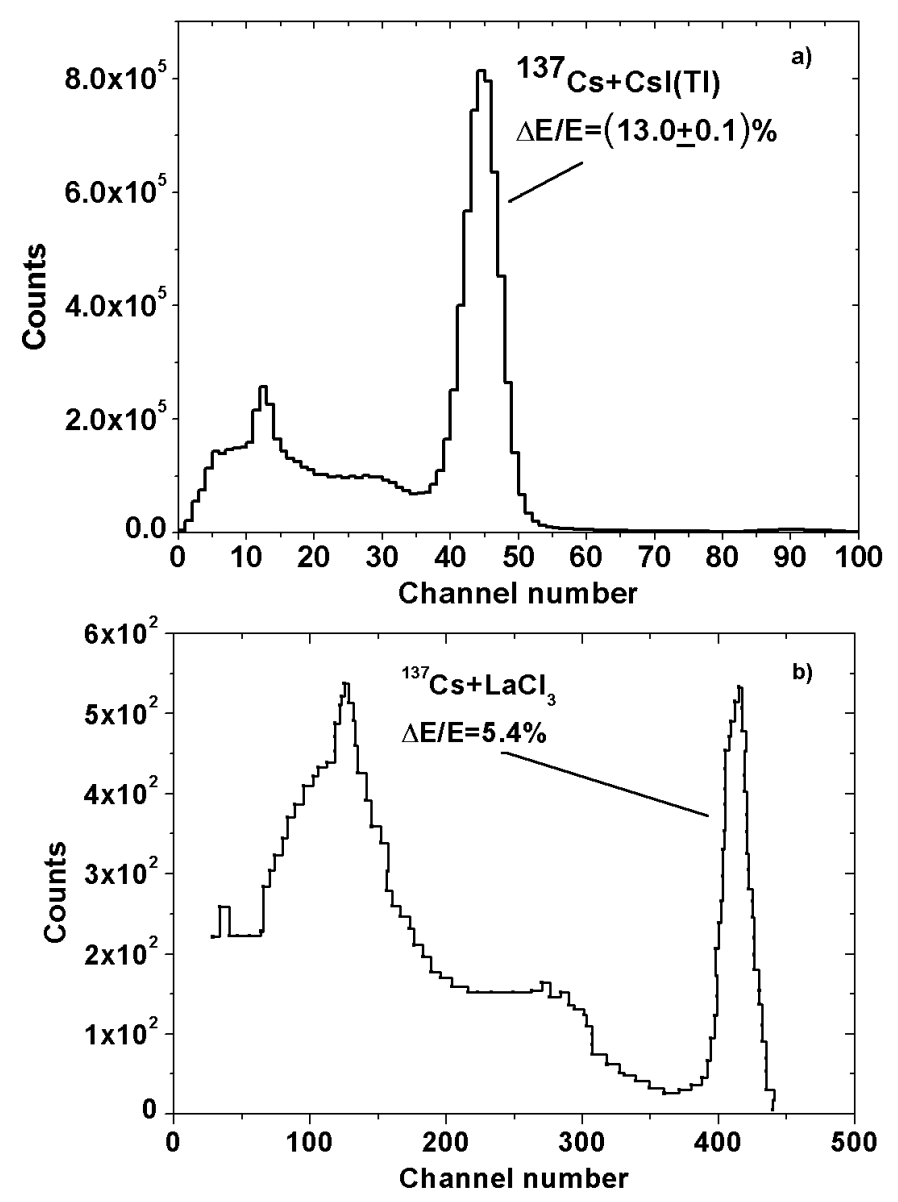

Fig. 5. The energy spectra from the radioactive source ${ }^{137} \mathrm{Cs}\left(\mathrm{E}_{\gamma}=662 \mathrm{keV}\right)$ measured a) by the combination of 3" $\times 3$ " CsI (Tl) crystal with PMT FEU-110 and b) by the combination of 3 " $\times 3$ " B350 crystal with PMT EMI9265-B53/FL (adopted from ${ }^{11}$ ).

can be explained by using the self mounting assembling of $\mathrm{LaCl}_{3}$ : Ce crystal with photomultiplier instead of manufacturer assembling.

Unfortunately all of the BrilLanCe series crystals has the week internal radioactivity due to ${ }^{227} \mathrm{Ac}(\gamma$-quanta with enrgies $323,329,338$ and $351 \mathrm{keV})$ and 138 $\mathrm{La}(\gamma$-quanta with enrgies 789 and $1436 \mathrm{keV})$ but the typical level of this activity is $\sim 2$ counts $/ \mathrm{s} / \mathrm{g}^{12}$ and it is too small for space born apparatus. Also such background could be used in the onboard inflight calibrations during satellite experiment similar to activation lines (for example, in $\mathrm{CsI}(\mathrm{Tl})$ crystal). This lines could be substracted during experiment using preflight calibrations (similar to internal line from ${ }^{40} \mathrm{~K}\left(\mathrm{E}_{\gamma}=1461 \mathrm{keV}\right)$ in the glass used in PMT and lightguide). 


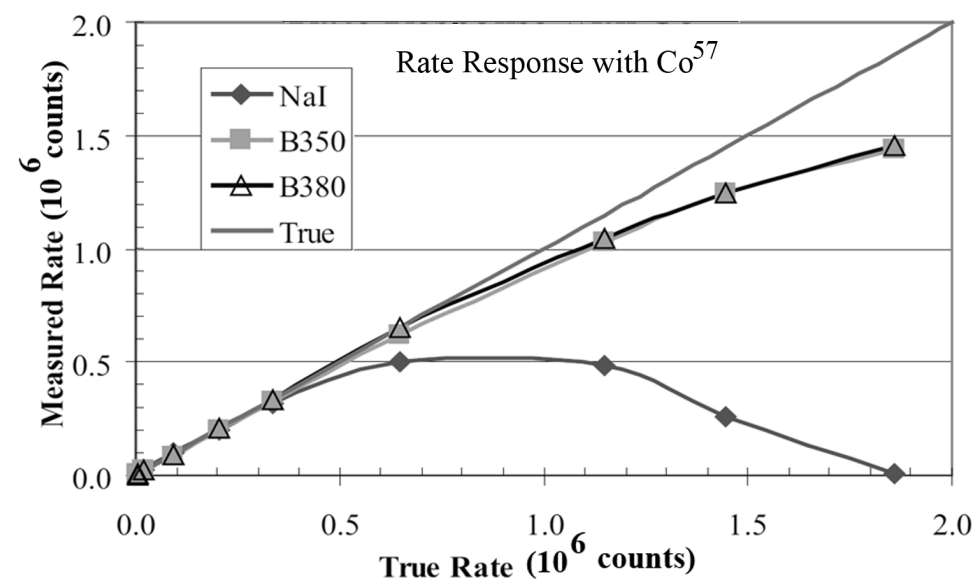

Fig. 6. The dependence of measured counts rate from real counts rate (adopted from ${ }^{11}$ ).

The BrilLanCe series scintillators pratically have not dependence of misplased counts on the activity of the mesuared source up to $1.5 \times 10^{6}$ counts $/ \mathrm{s}^{11}$. The dependence of measured counts rate from real counts rate is shown the Fig 6 . It is seen that more or less linearity region both for B350 and B380 crystals is $1 \div 1.5 \times 10^{6}$ counts/s and corresponding dynamic range of these scintillators is more than 6 magnitudes.

\section{Conclusions}

As we can see BrilLanCe crystals have got very best relative light output and energy resolution, highest linearity and excellent temperature stability. In the case of use BrilLanCe series crystal in DNP instead of $\mathrm{CsI}(\mathrm{Tl})$ we will obtain following advantages:

- energy resolution is better ( the factor of 2-3 ) hence low limit of detector energy band will be $\sim 20 \mathrm{keV}$ instead of $100 \mathrm{keV}$;

- temperature stability is better;

- light output components are faster (by the factor of the order) hence

- the time resolution will be $\sim 5 \mu$ s (instead of $1 \mathrm{~ms}$ ) for $\gamma$ up to $20 \mathrm{MeV}$, and $\sim 15 \mu \mathrm{s}$ (instead of $10 \mathrm{~ms}$ ) for events that need analysis of pulse shape $(\gamma$ and neutrons);

- possibility of registration of more intensive fluxes;

- no problem with fluorescence from high energy charged particles.

There are two possible opportunities of DNP in ZINA-NT experiment:

- $\mathrm{CsI}(\mathrm{Tl})$ : 
(a) energy band $100 \mathrm{keV}-300 \mathrm{MeV}$ ( $\gamma$-quanta);

(b) minimum time resolution $\sim 1 \mathrm{~ms}$;

(c) problem with high intensity events;

(d) problem with fluorescence from high energy charged particles;

- BrilLanCe350 $\left(\mathrm{LaCl}_{3}\right.$ : Ce $\left.(10 \% \mathrm{Ce})\right)$ :

(a) energy band $20 \mathrm{keV}-300 \mathrm{MeV}$ ( $\gamma$-quanta);

(b) minimum time resolution $\sim 5 \mu \mathrm{s}$;

(c) no problem with high intensity events;

(d) no problem with fluorescence from high energy charged particles.

Also we plane to use several parallel timescales: $\sim \mu$ s $($ or $\sim \mathrm{ms}$ in the dependence of used crystal), $1 \mathrm{~s}, 10 \mathrm{~s}, 64 \mathrm{~s}$ and $128 \mathrm{~s}$ (for events with low statistic -15.11 and $20.58 \mathrm{MeV}$ nuclear lines in solar flares, GRB extended emission and so on). Such set of timescales allows us more efficientally investigate events with strongly changing intensity.

\section{Acknowledgments}

The authors wish to thank prof. W.Q. Gan from Purple Mountain Observatory (China) for very useful discussions.

\section{References}

1. A. G. Batischev, A. M. Galper and S. A. Voronov et al, Instruments and Experimental Technicues 5 (1999) 25.

2. A. V. Bakaldin, A. G. Batischev and S. A. Voronov et al, Rossijskaya Akademiya Nauk, Izvestiya, Seriya Fizicheskaya 69 \# 6 (2005) 918.

3. A. I. Arkhangelskiy, A. G. Batischev and A. M. Galper et al, Proc. Scientific Session of the Moscow Engineering Physics Institute, Vol. 4 (MEPhI, Moscow, 2008) p. 74 (in Russian).

4. A. G. Batischev, S. V. Koldashev and P. Yu. Naumov et al, Proc. Scientific Session of the Moscow Engineering Physics Institute, Vol. 9 (MEPhI, Moscow, 2008), p. 167 (in Russian).

5. A. G. Batischev, S. V. Koldashev and A. A. Mednikov et al, in Proc. Scientific Session of the Moscow Engineering Physics Institute, Vol. 9 (MEPhI, Moscow, 2008), p. 169 (in Russian).

6. L. Varga, Nucl. Instr. and Meth. A 14 (1961) 254.

7. Saint-Gobain Technical Note, (www.detectors.saint-gobain.com, 2009).

8. C. Hoel, L. G. Sobotkaa and K. S. Shahb et al, Nucl. Instr. and Meth. A 540 (2005) 205.

9. F. C. L. Crespi, F. Camera and N. Blasi et al, Nucl. Instr. and Meth. A 602 (2009) 520.

10. M. Moszynski, A. Nassalskia and A. Syntfeld-Kazuch et al, Nucl. Instr. and Meth. A 568 (2006) 739.

11. W. Q. Gan, Private communications.

12. BrilLanCe 350 Product Data Sheet, (www.detectors.saint-gobain.com, 2010) 